\title{
Ronald Hartz
}

\section{Von anderen Organisationen - Ein Essay über Perspektiven kritischer Organisationsforschung ${ }^{1,2}$}

Critical Management Studies; Foucault; Genealogie; Heterotopie; immanente Kritik; kritische Organisationsforschung; Welterschließung

\section{Zusammenfassung}

Der Essay geht von der Beobachtung aus, dass trotz der Allgegenwart von Kritik die kritische Praxis sich auf einem notorisch schwankenden Grund befindet sowie angesichts propagierter gesellschaftlicher Alternativlosigkeiten und eines herrschenden zynischen Bewusstseins als ein eher naives und trübsinniges Geschäft erscheint. Mit Blick auf die Organisationsforschung werden deshalb mit der immanenten Kritik, der an Foucault anschließenden Genealogie sowie der welterschließenden Kritik drei Perspektiven kritischer Organisationsforschung diskutiert, welche der kritischen Praxis schärfere Konturen verleihen. Mit der welterschließenden Kritik, welche sich der Sichtbarmachung von Heterotopien, von anderen und alternativen Formen des Organisierens widmet, wird eine kritische Praxis vorgeschlagen, welche den Gegensatz von Affirmation und permanenter Kritik unterläuft und so zu einer Revitalisierung von Kritik beitragen kann.

\section{Schlüsselwörter}

Critical Management Studies · Foucault - Genealogie · Heterotopie · Immanente Kritik · Kritische Organisationsforschung · Welterschließung

\footnotetext{
${ }^{1}$ Preprint. Please do not quote without permission. Erschienen in: Managementforschung 27(1), S. 167191. https://doi.org/10.1365/s41113-017-0018-3

${ }^{2}$ Ich danke den Herausgebern der Managementforschung sowie den zwei anonymen GutachterInnen für die zahlreichen wertvollen und konstruktiven Hinweise. Eine frühere Fassung des Beitrags wurde im Kolloquium „Organisationspädagogik und CMS“ am Institut für Pädagogik der FAU Erlangen-Nürnberg im Mai 2015 sowie im Rahmen des Forums „Organisationsforschung“ des Instituts für interdisziplinäre Arbeitswissenschaft der Leibniz Universität Hannover im Mai 2016 vorgestellt. Auch hier möchte ich allen TeilnehmerInnen, insbesondere Nicolas Engel, Michael Göhlich, Christine Schwarz und Axel Haunschild, für die anregende und hilfreiche Diskussion danken.
} 


\begin{abstract}
The essay starts with the observation that, despite the omnipresence of criticism, critical practice is on a notoriously fluctuating ground. Even more the propagated lack of social alternatives and a dominant cynical consciousness turns critique to a rather naive and dull business. Regarding organizational research, the perspectives of immanent criticism, Foucault's genealogy, and critique as world-disclosure are discussed to give the critical practice sharper outlines. With world-disclosure devoted to the visualization of heterotopies, that is of alternative forms of organization, a critical practice is suggested which undermines the opposition between affirmation and permanent criticism, and thus contributes to a revitalization of critique.
\end{abstract}

Keywords

Critical Management Studies · Critical organization studies · Foucault - Genealogy · Heterotopia $\cdot$ Immanent critique $\cdot$ World-disclosure

\title{
Inhaltsübersicht
}

1. Einleitung - Kritik auf schwankendem Grund

2. Motive kritischer Praxis

3. Drei Einsatzpunkte kritischer Organisationsforschung

3.1. Entmythologisierung und Politisierung von Organisationen - kritische Organisationsforschung als immanente Kritik

3.2. Historisierung und De-Naturalisierung - kritische Organisationsforschung als genealogische Kritik

3.3. Kritische Organisationsforschung als welterschließende Kritik

3.3.1. Arbeit im Gelände - Rekonstruktion der Pluralität ökonomischer und organisationaler Formen

3.3.2. Jenseits des „Manufacturing Consent“ - Innerorganisationale Heterotopien als Vorschein alternativer Arbeits- und Organisationsformen

4. Résumé 


\section{Einleitung - Kritik auf schwankendem Grund}

Kritik ist ein grundlegender, wenn auch nicht immer zur Artikulation gebrachter Begleiter menschlichen Handelns. Jede Form des Beurteilens menschlichen Handelns, sozialer Zustände, gesellschaftlicher Institutionen oder Werte, jede Einschätzung einer organisationalen Praxis als gut, falsch, angemessen oder problematisch ist in dieser grundlegenden Hinsicht Kritik. Dies gilt konstitutiv im lebensweltlichen Zusammenhang, für den Bereich der Medien, für die politische Praxis und last not least auch für organisationale Praktiken. Letzteres wird prominent sichtbar an den öffentlichen, politischen und medialen Diskursen über Geschäftspraktiken in Konzernen wie beispielhaft Volkswagen, Shell, Enron, der Deutschen Bank oder Monsanto. Überdeutlich zeigt sich dies auch angesichts von Katastrophen wie der Explosion der Ölplattform Deep Water Horizon im April 2010, dem Chemieunfall im indischen Bhopal 1984 oder des Einsturzes des Fabrikgebäudes Rana Plaza in Bangladesch im Jahr 2013 und den sich hieran anschließenden Kontroversen über die soziale und ökologische Verantwortung von Unternehmen und damit verbundenen zivilgesellschaftlichen Mobilisierungen.

Der folgende Essay verfolgt vor diesem Hintergrund das Ziel, der Idee und dem Projekt einer kritischen Organisationsforschung durch die Diskussion unterschiedlicher Formen und Einsatzpunkte von Kritik eine schärfere Kontur zu verleihen. Das diese Konturierung notwendig ist, ergibt sich erstens aus der grundlegenden Einsicht, dass Kritik sich auf schwankendem Grund befindet oder sich in notorisch schwierig zu befahrenden Gewässern bewegt und - so mag es bezogen auf den akademischen Diskurs manchmal erscheinen - dort eher vor sich hindümpelt. Für diese Situation sollen zunächst in dieser Einleitung einige Gründe benannt werden. Ausgehend von der Diskussion grundlegender Motive kritischer Praxis, soll zweitens dieser Erschöpfung der Kritik begegnet werden, indem mit der immanenten, genealogischen und welterschließenden Kritik drei Einsatzpunkte kritischer Organisationsforschung diskutiert werden. Die klassische Form der immanenten Kritik und die an Foucault anschließende Perspektive der Genealogie zielen auf kritische Vermessungen organisationaler Realitäten zwischen Anspruch und Wirklichkeit (immanente Kritik) und auf die Rekonstruktion von Organisation als historisch kontingenter Regierungsform der Moderne (Genealogie). Mit der welterschließenden Kritik wird anschließend eine Kritikform vorgeschlagen, welche den Blick auf Heterotopien - auf ,andere Organisationen“ und alternative Organisationsformen lenkt und eine Kritik ermöglicht, welche zwischen Affirmation und kritischer Reflexion von Organisationen oszilliert und das Erbe immanenter 
und genealogischer Kritik in sich aufnehmen kann. Mit der welterschließenden Kritik ergibt sich ein Einsatzpunkt kritischer Organisationsforschung, welches einen dritten Weg zwischen den Alternativen permanenter Kritik und Affirmation eröffnet und somit $\mathrm{zu}$ einer Revitalisierung kritischer Praxis beitragen kann. Methodische Fragen als auch konkrete Vorschläge für empirische Projekte bleiben im vorliegenden Essay dabei weitgehend ausgespart. Beides bedürfte einer weiteren ausführlichen Betrachtung (vgl. die Hinweise bei Wolf (1999, S. 187) zur „methodischen Entdogmatisierung“ sowie Alvesson/Deetz 2000; Flyvbjerg 2001, 2003; Gibson-Graham 2008; Alvesson/Ashcraft 2009).

Dafür, dass Kritik sich auf schwankendem Grund befindet, lassen sich nun zunächst eine Reihe von Gründen angeben. In historischer Perspektive lässt sich erstens von einer sozialen Generalisierung von Kritik und der Verwendung des Kritikbegriffs in der Moderne, wenn nicht sogar von einer Verpflichtung zur Kritik sprechen (Röttgers 2004, S. 674-675). Dabei wird Kritik zugleich bagatellisiert: „,[D]ass man sich allgemein kritisch nennt, hindert nicht, dass radikale Kritik wie eh und je ebenso allgemein suspekt erscheint" (Röttgers 2004, S. 675). Beinahe ist heute verdächtig, wer nichts kritisiert. Klammert man diese Bagatellisierung und auch Ritualisierung von Kritik zunächst ein, verweist Kritik als Bestandteil menschlicher Praxis immer auf Spielräume menschlichen und organisationalen Handelns, auf andere Möglichkeiten der Interpretation und der Bedeutungszuweisung gesellschaftlicher Praxis Praxis kann so, aber auch anders möglich sein. Damit wird ein weiteres Problem von Kritik sichtbar: Was, wenn scheinbar keine gesellschaftlichen oder organisationalen Alternativen zur Verhandlung stehen? Kritik, akademische Kritik zumal, mag die Kontingenz und Offenheit des Sozialen betonen und, etwa in poststrukturalistischer Perspektive, Institutionen und Organisationen „in permanenter Bewegung“ sehen, welche „fortlaufenden Transformationen, Verschiebungen und Brüchen“ (Kneer 2008, S. 129) unterliegen. Diese Offenheit scheint jedoch erst Überzeugungskraft im Zusammenhang mit krisenhaften gesellschaftlichen Ereignissen und Problematisierungen $\mathrm{zu}$ gewinnen, wie dies im Kontext der globalen Finanzkrise 2008-09 zu beobachten war (Morgan et al. 2011; Zanoni et al. 2017). Zugleich ist die Geschichte solcher Ereignisse auch die Geschichte enttäuschter Erwartungen und der Erneuerung der Propagierung der Alternativlosigkeit - wie sich im Fall der globalen Finanzkrise und der sich anschließenden Euro- und Griechenlandkrise exemplarisch besichtigen lässt (Morgan 2011; Crouch 2011). Damit öffnet sich ein dritter Problembereich der aktuellen Verfasstheit von Kritik, welchen man als die Entwertung herrschaftskritischer, auch auf die Totalität der Gesellschaft zielender Kritik bezeichnen kann. Der Verweis auf gesellschaftliche Strukturen, soziale Ungleichheiten und Diskriminierung - mithin darauf was 
mit Johan Galtung (1969) „strukturelle Gewalt“ genannt werden kann - wird ob des herrschenden Diktums, das ,jeder [sic!] seines Glückes Schmied“ sei, oftmals entweder ungläubig aufgenommen, als persönliche Beleidigung verstanden oder als notorischer Kritizismus psychologisiert. Mehr noch aber begegnet dem machtkritischen Verweis auf gesellschaftliche Ungleichheiten und Diskriminierungen ein vollends aufgeklärtes Bewusstsein, eine zynische Vernunft (Sloterdijk 1983), welche um all die Ungerechtigkeiten augenzwinkernd Bescheid weiß, aber das Spiel dennoch mitspielt: ,[E]ven if we do not things seriously, even if we keep an ironical distance, we are still doing them“ (Žižek 1989, S. 30). Im organisationalen Kontext erweisen sich Ironie und Zynismus dann als Mechanismen der Aufrechterhaltung organisationaler (Macht-)Verhältnisse, da diese den Akteuren die Illusion der Freiheit und der Distanzierung vom Betrieb ermöglicht (Fleming/Spicer 2003). Kritik erscheint dann auch hier als ein eher naives Unterfangen.

Kritik mag also ein notorischer Begleiter menschlichen Handelns sein. Gleichwohl sind die eben genannten Punkte wenig ermutigend und führen einen durchaus depressiven Grundton mit sich, in welcher Kritik angesichts der Verhältnisse eher mit der Melancholie verschwistert zu sein scheint. Das Kritik sich zudem epistemologisch auf schwankendem Grund befindet, ist auch für die Organisationsforschung kein neuer Befund. So fand etwa in den 1990er Jahren eine durchaus hitzige Debatte um das Verhältnis von Postmoderne und Organisationsforschung statt. Das „Ende der großen Erzählungen“ von Emanzipation und Erkenntnis (Lyotard 1986) und die Zurückweisung universeller Wahrheitsansprüche fanden ein breites Echo in der Organisationsforschung (Cooper/Burrell 1988; Hassard/Parker 1993). Für Martin Parker (1993, S. 205) führte diese Debatte auch zu der Frage, warum man überhaupt schreiben solle: „Postmodernists might be correct about the dangers of assuming that I write the truth but they do not give me a clear reason for wanting to write at all - and I do“. Insofern ist ein schwankender Boden zunächst kein Grund zur Resignation und für ein Verstummen, sondern zieht eine notwendige reflexive Dimension ein, welche die Möglichkeiten der Kritik neu ausbuchstabieren hilft. In dieser reflexiven Absicht soll im Folgenden dieser schwankende Grund zunächst danach befragt und vermessen werden, welche Ausgangspunkte und Motive Kritik anleiten können. Im Hauptteil des Beitrages werden hieran anschließend immanente, genealogische und welterschließende Kritik als Einsatzpunkte kritischer Organisationsforschung diskutiert. Ein Résumé beschließt den Essay. 


\section{Motive kritischer Praxis}

Im Kontext der Critical Management Studies (CMS) werden immer wieder eine Reihe von problematischen und „dunklen“ Seiten moderner Organisationen und modernen Managements angeführt, um die Notwendigkeit einer kritischen Management- und Organisationsforschung zu begründen. So heißt es exemplarisch bei Alvesson, Bridgman und Willmott (2009, S. 8): ,[I]nstead of enabling human flourishing, organizations incubate and normalize stress and bad health, naturalize subordination and exploitation, demand conformism, inhibit free communication, erode morality, create and reinforce ethic and gender inequalities, and so on“. Für Adler, Forbes und Willmott (2007, S. 119) ist der Ausgangspunkt der CMS eine grundsätzliche und tiefsitzende Skepsis hinsichtlich der „moral defensibility and the social and ecological sustainability of prevailing conceptions and forms of management and organization“.

Diese beispielhaften „Spieleröffnungen“ sind als Aufzählungen und Beschreibungen suggestiv. Sie setzen einen Rahmen für die CMS, welcher alles andere als schwankend erscheint. Diesen umfassenden Problematisierungen von Organisation und Management kann man folgen oder aufgrund des forcierten Tones sich bereits an diesem Punkt abwenden. Jenseits dieser zwei Reaktionen und jenseits konkreter organisationaler Analysen bleibt in beiden Spieleröffnungen vage, warum - auch wenn man die „dunklen Seiten“ von Organisationen anerkennt - es nun einer kritischen Managementforschung bedarf. Vereinfacht ließe sich immer noch fragen: „So what?“. Es lohnt sich insofern, sich näher mit einigen Motiven und Ausgangspunkten kritischer Praxis auseinanderzusetzen, welche über die CMS hinausgreifen. Im Folgenden sollen Leiden, Krise und „Notwendigkeiten der Gegenwart" als drei wichtige Motive näher betrachtet werden.

Tilo Wesche (2009, S. 201) spricht davon, dass „[d]er Ausgangspunkt jeder Kritik ... die Diagnose einer Wirklichkeit [bildet], die nicht sein soll: eines Leidens, an dem Anstoß genommen; eines Unrechts, an dem sich Widerspruch entzündet; eines Elends, das empört“. Auch Michael Walzer (2000, S. 714) postuliert einen starken Zusammenhang zwischen der Empfindung eines Leidens und Kritik: ,[J]emand der das Leiden der Opfer nicht einräumt, [wird] gar nichts zur Kritik beizutragen haben“. Adorno (1966, S. 29) spricht schließlich davon, dass, solange die Gesellschaft Angst und damit objektiv Leiden erzeugt, „das Bedürfnis, Leiden beredt werden zu lassen [...] Bedingung aller Wahrheit“ sei. Eine kritische Soziologie gewänne ihr Motiv aus der „konkreten Denunziation des Unmenschlichen“ (Adorno 2010, S. 261), der Konfrontation des Leidens mit der Möglichkeit ihrer Beseitigung. 
In anderer Diktion ist für Hartmut Rosa (2009, S. 24) die Frage nach den sozialen Bedingungen, unter denen gelingendes menschliches Leben möglich ist oder vereitelt wird, der eigentliche „Humus“ einer sich kritisch verstehenden Soziologie. Empathie, die Anerkennung des Leidens Anderer, die Konstatierung eines Unrechts erscheint somit als ein basaler Moment für ein Bedürfnis nach Kritik. Anders formuliert: Wer nichts empfindet angesichts der Opfer in einem eingestürzten Fabrikgebäude in Bangladesch oder der massiven Umweltkatastrophe im Golf von Mexiko nach der Explosion von Deep Water Horizon, wird schwerlich einen Wunsch nach Kritik verspüren. Der Zusammenhang von Leid und Kritik ist nun jedoch kein unmittelbarer, worauf Susan Sontag (2003) in ihrem Essay Das Leiden anderer betrachten eindrücklich hinweist: „Wo es um das Betrachten des Leidens anderer geht, sollte man kein >Wir als selbstverständlich voraussetzen“" (Sontag 2003, S. 13). Ein erstes Problem besteht in der im Normalfall grundsätzlichen medialen Vermitteltheit von Ereignissen. Hier ist man zunächst damit konfrontiert, dass Leiden entweder überhaupt nicht in den Bereich der Sichtbarkeit, in den Bereich der Artikulation gebracht wird oder Gegenstand diskursiver Umdeutungen ist. Ein weiteres Problem ist, dass die Artikulation von Leid oftmals in einer ikonischen Verdichtung erfolgt, welche nicht notwendig zur Kritik, sondern zur passiven Hinnahme führt: „Quälende Fotos verlieren nicht unbedingt die Kraft zu schockieren. Aber wenn es darum geht, etwas zu begreifen, helfen sie kaum weiter“ (Sontag 2003, S. 104). Dass durch die Bilder, Texte oder andere Medien erzeugte Mitgefühl ist somit höchst ambivalent. So kann dieses einerseits den Impuls für Kritik liefern, wie dies beispielhaft in einigen Arbeiten Foucaults, so in Der Fall Rivière (Foucault 1975), der Studie über den Fall des Hermaphroditen Alexina/Herculine Barbin (Foucault 1998) oder in Das Leben der infamen Menschen (Foucault 2005a) sichtbar wird. In Das Leben der infamen Menschen heißt es: ,[I]ch gestehe, dass diese >Novellen $<$ [...] mich tiefer im Mark erschüttert haben als das, was man gewöhnlich die Literatur nennt“" (Foucault 2005a, S. 311). In Der Fall Rivière heißt es ganz ähnlich: „Der Ausgangspunkt der ganzen Arbeit war unsere Betroffenheit“ (Foucault 1975, S. 9). Andererseits kann Mitgefühl zu Passivität und (durchaus lustvoller) Ohnmacht führen und enthebt von der Empfindung der Komplizenschaft. Die Grenze zwischen der Darstellung von Leiden und von dessen Ästhetisierung als Kitsch, welche in Form einer Emotionalisierung der Massen und der Konstruktion einer vermeintlich globalen Humanität, eines „universellen Menschseins“ Kritik eher stillstellt denn befördert (Linstead 2002), ist damit fließend. In anderer Diktion formulieren Horkheimer und Adorno: „Das Hinstarren aufs Unheil hat etwas von Faszination. Damit aber etwas vom geheimen Einverständnis“ (1988, S. 245). Leiden verliert insofern seine unmittelbare 
Selbstverständlichkeit als Ausgangspunkt der Kritik und die Darstellung und Artikulation von Leiden und Unrecht darf nicht in einem vor-reflexiven Raum verbleiben. Gleichwohl: Die Anerkennung von Leid stellt nach wie vor einen basalen Impuls für eine kritische Praxis dar. Dies zeigt sich ex negativo insbesondere an den Versuchen, Leiden gerade nicht beredt werden zu lassen, dieses umzudeuten oder in Kitsch aufgehen zu lassen.

Einen zweiten Ausgangspunkt von Kritik stellen gesellschaftliche Krisenereignisse dar. Betrachtet man exemplarisch die globale Finanzkrise 2008-09, zeigt sich im akademischen Bereich im Rückblick eine Zunahme von Reflexivität, welche das eigene Handeln, das eigene wissenschaftliche Arbeiten einer kritischen Betrachtung unterzog. Die letzten Tagungen der CMS waren hiervon geprägt und die Wissenschaftskommission Organisation im Verband der Hochschullehrer der BWL tagte im Februar 2009 unter dem auch selbstkritischen Titel: „Finanzkrise - auch eine Krise der BWL/Organisationsforschung?“ Auch in historischer Perspektive zeigt sich ein enger Zusammenhang von gesellschaftlicher Krisendiagnostik und der Artikulation von Kritik (Koselleck 2010, S. 203ff.). Bezogen auf die Krisen der Gegenwart befindet sich für die CMS die tradierte Managementforschung nicht nur in einer epistemologischen, sondern auch in einer moralischen Krise (Fournier und Grey 2000; Parker 2002a; Ghoshal 2005; Marens 2010). Der Zusammenhang von Kritik und Krise ist nun in zumindest zweierlei Hinsicht problembehaftet. Erstens erweist sich der Zeitkern der Krisendiagnostik als Schwierigkeit: Über die Krise der BWL/Organisationsforschung nachzudenken, blieb dann doch ein recht singuläres Ereignis. Ein zweites, bedeutsameres Moment ist die diskursive Normalisierung von Krisenereignissen im ökonomischen als auch organisationalen Kontext, wie es exemplarisch in der diskursiven Bearbeitung der globalen Finanzkrise sichtbar wurde (Hartz 2012, 2013; Koselleck 2010, S. 210ff.). Das sich hierbei abzeichnende Krisennarrativ wurde dominiert durch die Semantik der schöpferischen Zerstörung und der Notwendigkeit des Scheiterns, welche das durch die Krise induzierte Leiden auf lange Sicht als notwendig und damit gerechtfertigt ansah. Kritik erwächst somit immer wieder aus der Diagnose einer Krisenhaftigkeit, muss sich jedoch reflexiv mit dem Status und der diskursiven Bearbeitung von Krisen auseinandersetzen. Im ökonomischen und organisationalen Kontext heißt dies insbesondere, skeptische Distanz gegenüber der Deutung und metaphysischen Aufwertung von Krisen als Momenten schöpferischer Zerstörung und dem damit verknüpften Fortschritts- und Rationalitätsnarrativ zu wahren.

Während Leiden und Krise auf bestimmte gesellschaftliche Ereignisse oder soziale Zustände verweisen und Motive für einen konkreten Einsatz von Kritik darstellen, lässt sich drittens, in 
Anlehnung an die Arbeiten Michel Foucaults, von den „Notwendigkeiten der Gegenwart“ (Sarasin 2006, S. 6) sprechen, welche einen Ausgangspunkt des Schreibens und der Kritik darstellen können. Siegfried Jäger formuliert ähnlich allgemein, wenn er von ,gesellschaftlich brisanten Themen“ (2012, S. 151) als Einsatzpunkt kritischer Analysen spricht. Was als „brisant“ oder „,notwendig“ erscheint, hängt erstens mit der eigenen Involviertheit in aktuelle gesellschaftliche Probleme zusammen (bei Foucault unter anderem sichtbar in dessen eigenen Bezügen zur Psychiatrie oder zu den Gefängnisunruhen) und zweitens damit, was auch, über den konkreten Bezug zu Formen des Leidens und zu Krisenereignissen hinaus, in fortgesetzter Reflexion als ein Drängeln der Gegenwart wahrgenommen wird. Die Frage nach den „Notwendigkeiten der Gegenwart“ kann als Korrektiv wirken, da diese es erlaubt, eine kritische Distanz zu den Moden des wissenschaftlichen Feldes als auch zu den hochgradigen Spezialisierungen und spezifischen Publikationsanforderungen im Feld einzunehmen. Die Skepsis gegenüber der „moral defensibility and the social and ecological sustainability of prevailing conceptions and forms of management and organization“ (Adler et al. 2007) kann eine solche Notwendigkeit begründen, welche kritische Analysen zu rahmen hilft. Diese Notwendigkeiten führen dann immer auch die Frage mit sich, wie es denn anders, wie es denn besser sein könnte. Die kritische Management- und Organisationsforschung lässt sich dann als „progressive management scholarship“ (Adler 2016) fortschreiben und neu positionieren. Kritik wird dann zu einem Modus der Welterschließung (vgl. Abschnitt 3.3), sowohl was die Frage der Alternativen in der Vergangenheit, in der Gegenwart als auch die möglichen Zukünfte von Ökonomie und Organisation als „alternative economic futures“ (Adler 2016) betrifft.

Leiden, Krise und Notwendigkeiten der Gegenwart bezeichnen drei unterschiedliche gesellschaftliche und historische Konstellationen und unterscheidbare Ausgangspunkte der Kritik. Diese Motive können die Impulse für eine Kritik an gesellschaftlichen oder organisationalen Verhältnissen liefern. Gleichwohl wird sichtbar wie vermittelt, prekär und umkämpft diese Ausgangspunkte sein können. Hier erscheint nichts voraussetzungsfrei und einfach gegeben. Kritik muss insofern hier bereits als Diskurskritik eine reflexive Dimension einziehen - was gelangt nicht zur Artikulation, was wird der Stille überantwortet? Was gelangt zur Artikulation, um Empörung und eine mögliche Artikulation von Kritik stillzustellen? Sichtbar wird auch, dass der eigene Erfahrungshintergrund, das eigene wissenschaftliche Feld und die subjektive, wiederum vielfältig vermittelte Wahrnehmung gesellschaftlicher Zusammenhänge und Ereignisse für die Artikulation von Kritik bedeutsam sind. Insofern bewegt sich auch hier kritische Praxis auf schwankendem Grund und bedarf 
entsprechender Selbstreflexion. Für das akademische Feld der Organisationsforschung bedeutet dies wiederum, sich mit den diskursiven und institutionellen Mechanismen auseinandersetzen, welche Themen entweder als relevant und forschungsbedürftig oder als ephemer, marginal oder als jenseits des eigenen Faches und der eigenen Kompetenz liegend rahmt.

\section{Drei Einsatzpunkte kritischer Organisationsforschung}

Vor dem Hintergrund des - wie weiter oben dargelegt - Insistierens der CMS auf die negativen und problematischen Effekte managerialer und organisationaler Praktiken, stellt für Fournier und Grey (2000, S. 16) eine Art „Unbehagen“ den Ausgangspunkt der CMS dar: „[T] here is something wrong with management as a practice and as a body of knowledge“ (ebd.). Dieses diffuse Bild aufnehmend, verweist ein Unbehagen zunächst auf den Raum des Vorsprachlichen. So existiert für Bormann (1973, Sp. 809) „[i]n der Tat ... schon ein bestimmtes sprachloses Handeln, welches wir als Kritik empfinden, ein Gefühl der Unstimmigkeit und einen Ausdruck des Missfallens, die sich auf vielerlei Weise kundgeben können“. Gelangt nun dieses Unbehagen in der Form der Kritik zur Artikulation, lassen sich im Sinne einer Selbstreflexion der kritischen Praxis eine Reihe von Fragestellungen und Problematisierungen festhalten (vgl. Jaeggi/Wesche 2009, S. 8-9):

- Wie ist das Verhältnis zwischen der Kritik des Bestehenden zur Möglichkeit der Veränderung und des Neuen? Muss Kritik konstruktiv werden, um letztlich auch Wirksamkeit zu entfalten (Adorno 1977)?

- Was sind die Maßstäbe der Kritik? Verbleibt sie intern und rekurriert auf existierende Normen, nimmt diese einen externen, sich etwa auf universale Werte berufenden Standpunkt ein (Geuss 1983; Celikates 2009) oder geht es ihr um die Destabilisierung und Subversion existierender Praktiken (Butler 1991, 2009)?

- Bedarf es der Nähe zum kritisierten Gegenstand oder ist Distanz die Bedingung kritischer Praxis (Walzer 2000)?

- Wie ist schließlich das Verhältnis von Theorie und kritischer Praxis zu bestimmen, welches auch die Frage nach der Deutungsmacht und Deutungshoheit wissenschaftlicher Analyse und/oder von sozialen Akteuren betrifft (Celikates 2009)? 
Diese Problematisierungen stehen im Hintergrund der folgenden Darstellung der Einsatzpunkte kritischer Organisationsforschung und spielen an verschiedenen Stellen eine zumindest implizite Rolle. Die Frage der Rechtfertigung von Kritik dabei nicht ins Zentrum zu stellen, erscheint uns insofern und zunächst selbst gerechtfertigt, da auch für die kritische Management- und Organisationsforschung die Gefahr der Selbstbeschäftigung virulent ist (Cooke 2008). Ein zu viel an Bescheidenheit läuft letztlich auf Adornos Feststellung hinaus, dass objektiv „der introvertierte Gedankenarchitekt ... hinter dem Mond [wohnt], den die extrovertierten Techniker beschlagnahmen“ (Adorno 1966, S. 15).

In Abgrenzung zu dieser Selbstbeschäftigung soll ein pragmatisch motivierter Vorschlag unterbreitet werden, indem unterschiedliche Einsatzpunkte kritischer Organisationsforschung skizziert werden, welche durch unterschiedliche Stoßrichtungen und Reichweiten der Kritik gekennzeichnet sind. In zugespitzter Hinsicht lässt sich dabei auch von Stufen kritischer Organisationsforschung sprechen.

\subsection{Entmythologisierung und Politisierung von Organisationen - kritische Organisationsforschung als immanente Kritik}

Kritische Organisationsforschung lässt sich zunächst in den Zusammenhang der Idee der immanenten Kritik stellen (Adorno 1977; Geuss 1983; Romero 2014). Immanente Kritik entnimmt die normativen Maßstäbe ihrer Kritik aus der sozialen Realität, und lässt sich von einer Standpunktkritik unterscheiden, welche ihre Maßstäbe der Kritik von außerhalb des kritisierten Gegenstandes nimmt. Immanente Kritik konfrontiert die „Wirklichkeiten mit den Normen ..., auf welche jene Wirklichkeiten sich berufen“"(Adorno 1977, S. 792). Sie nimmt die vorhandenen und artikulierten Normen, Werte, Versprechungen, Rationalitäten ernst und stellt die Frage nach deren Realisierung. Die Diskrepanz zwischen Anspruch und Wirklichkeit wird zum Gegenstand der Kritik, wie es Adorno mit Bezug auf Ideologiekritik als immanenter Kritik formuliert: „Unwahr werden eigentliche Ideologien erst durch ihr Verhältnis zu der bestehenden Wirklichkeit. Sie können san sich` wahr sein, so wie die Ideen Freiheit, Menschlichkeit, Gerechtigkeit es sind, aber sie gebärden sich, als wären sie bereits realisiert“ (Adorno 1954, S. 473).

Eine kritische Organisationsforschung, welche der Idee der immanenten Kritik folgt, kann sich zunächst auf ein reiches Erbe einer kritischen Reflexion organisationalen Geschehens zwischen Anspruch und Wirklichkeit stützen, welches in seinen unterschiedlichen Formen 
nicht immer notwendig den Begriff der Kritik stark macht bzw. diesen für sich selbst in Anspruch nimmt. Die hier zu verorteten Arbeiten tragen zu einer „Entmythologisierung von Organisationen“ bei, wie es Klaus Türk (1989, S. 30) in Anlehnung an Benson (1983) einmal mit Bezug auf die frühen Arbeiten des Neoinstitutionalismus formuliert hat. Diese Entmythologisierung befragt - vereinfacht gesprochen - Organisationen auf deren eigene Rationalität hin. Dieses in den Blick zu nehmende Erbe ist dann in Teilen selbst bestimmt durch die Suche nach Rationalität, nach Effizienz oder dem one best way und insofern auch in ihrem Scheitern lehrreich: Sie brachen auf ,um den heiligen Gral zu finden, und kehrten heim mit einer zerbrochenen Teetasse" - so etwa das Resümee William H. Starbucks zu den einflussreichen Arbeiten der Aston-Gruppe (1981, S. 193, zitiert nach Ortmann 2008, S. 289). Einige weitere ausgewählte Bezüge aus dem Feld der Organisationsforschung seien hier angeführt, ohne dem Anspruch nach hier erschöpfend sein zu können. Da wäre etwa Chester Barnards 1938 erschienenes Werk The Functions of the Executive (Barnard 1968), Klassiker eines realistischen Blicks auf organisationales Handeln und organisationale Macht. Von Barnard stammt auch der Begriff der „Organisationspersönlichkeit“ und Günther Ortmann (Ortmann et al. 1997, S. 341; Ortmann 2008, S. 290-291) greift wiederholt die von Barnard erzählte Anekdote über eine Telefonistin der New Jersey Bell Telephone Company auf, welche eine nicht sehr attraktive Stelle in einem Außenbezirk annahm, um während der Arbeit das Haus ihrer kranken Mutter beobachten zu können. Als das Haus eines Tages Feuer fing, blieb die Telefonistin an ihrem Arbeitsplatz und sah mit an, wie das Haus niederbrannte. Für Barnard ein ausgezeichnetes Beispiel einer „Organisationspersönlichkeit“, welche ihre individuellen Bedürfnisse zurückstellte und „,außerordentlichen moralischen Mut“ bewies. Solcherart Geschichten sind es, welche kritisch gewendet danach fragen lassen, welche subjektiven Deformationen in Organisationen stattfinden und befördert werden. Die von Klaus Türk (1997, S. 172) kritisch diskutierte „Formkorrespondenz zwischen Organisationsform und Subjektform“ verweist auch ,auf die Restringiertheit und Pathologie besonders ,gut‘ angepasster Mentalitäten“ (ebd., S. 173, vgl. hierzu auch Adorno 1953), mithin auf das Leiden in und an Organisationen und das Leid, was im extremen Fall, Türk verweist hierbei auf Eichmann, von diesen gut angepassten Mentalitäten ausgehen kann.

Wenngleich im Bezug zum gerade Ausgeführten einen harten Schnitt markierend, sind jene im Garbage Can Ansatz formulierten Einsichten über die Ambiguitäten, Irrationalitäten und die machtdurchsetzte Kontingenz organisationaler Entscheidungsprozesse als Form immanenter Kritik instruktiv. In der nüchternen Diktion von Michael Cohen, James G. March und Johan P. Olsen (1972, S. 2): „,[A]n organization is a collection of choices looking for 
problems, issues and feelings looking for decision situations in which they might be aired, solutions looking for issues to which they might be the answer, and decisions makers looking for work“. Michel Crozier und Erhard Friedberg (1979) verweisen auf den machtdurchsetzten Charakter von Organisationen und darauf, dass es eben jene Machtspiele sind, welche zugleich den Fortbestand von Organisationen sichern. Organisationen werden hier vollends zu politischen Gebilden. Schließlich John W. Meyer und Brian Rowan, welche die simple Frage stellen, warum bestimmte formale Strukturen sich in endemischer Form in modernen Organisationen verbreitet haben und deren Antwort - formale Strukturen als „Myth and Cerenomy“, welche gesellschaftlichen Rationalitätsmythen folgen - immer noch ein kritischer Stachel gegen simplifizierende Vorstellungen ist, dass „formal structures actually coordinate and control work“ (Meyer/Rowan 1977, S. 343). Für Nils Brunsson (1989) sind Heuchelei und Scheinheiligkeit konstitutiv für Organisationen, insofern diese den Graben von Ideen und Handeln überbrücken und widersprüchliche Erwartungen befriedigen helfen. Dabei bleibt es nicht bei einer Entkopplung, vielmehr sieht Brunsson eine umgekehrte Kopplung am Werk: Je mehr die Meere verseucht werden, umso mehr sehen wir die Inszenierung von Umweltschutzkampagnen; je fragwürdiger die Arbeitsverhältnisse in der Textilindustrie, umso stärker das artikulierte Engagement der agierenden Unternehmen etc. pp. Auch die zahlreichen Studien, welche sich in den letzten Jahren der „dark side of organization“ zugewendet haben (Griffin/O’Leary-Kelly 2004; Vaughan 1999; Linstead et al. 2014), lassen sich in den Zusammenhang immanenter Kritik stellen. Für Griffin und O’Leary-Kelly (2004, S. Xv) bezeichnet und umfasst diese dunkle Seite von Organisationen ,situations in which people hurt other people, injustices are perpetuated and magnified, and the pursuits of wealth, power or revenge lead people to behaviours that others can only see as unethical, illegal, despicable, or reprehensible“ (Griffin/O’Leary-Kelly, 2004, S. xv). Als Form immanenter Kritik ist diese dunkle Seite ,not a dark out-side, a phenomenon 'out there' that impinges upon organizational action, but a dark interior to be found within organizational boundaries and practice“ (Linstead et al. 2014, S. 166).

Diese hier nur exemplarisch angeführten Studien mehren die Zweifel an der Rationalität von Organisationen, stellen die Frage nach der Legitimation organisationaler Verhältnisse, rücken im Fall der „dunklen Seite“ konkretes Leiden an und in Organisationen in den Mittelpunkt oder geben Organisationen durchaus auch einmal der Lächerlichkeit preis. Zugleich verbleibt die Kritik an den Normen der Organisation im Hinblick auf Rationalität, Effizienz oder der Realisierung des Beschäftigtenwohls orientiert, gewinnt aber hierdurch sowohl ihren kritischen Stachel als auch an Stärke und Plastizität. Sie verweist auf Deformationen, 
Pathologien, Macht, Irrationalitäten im Herzen der vermeintlich herrschenden Rationalität und gibt in den besten Fällen den Blick auf organisationale Ursachen von Frustration und Leiden frei. Die Frage, ob Organisation denn auch anders sein könne, lauert zwar auf der Rückseite der jeweiligen Befunde, gleichwohl verbleibt immanente Kritik in den selbstgesetzten Schranken der Immanenz. Die im Folgenden diskutierte genealogische Kritik als zweiter möglicher Einsatzpunkt kritischer Organisationsforschung erweitert den Blick auf die historische Gewordenheit von Organisationen in einer dezidiert machtkritischen Perspektive.

\subsection{Historisierung und Denaturalisierung - kritische Organisationsforschung als genealogische Kritik}

Kritische Organisationsforschung als genealogische Kritik unterwirft das organisationale SoSein einer historischen Kritik, nimmt also die Dimension der Geschichte systematisch in den Kritikzusammenhang auf. Genealogische Kritiken lösen zugleich „,einen Blick- und Perspektivenwechsel aus, nach dem das, wovon sie handeln (die >moderneく Moral, das >moderne Strafsystem) nicht mehr so gesehen werden kann wie zuvor“" (Saar 2009, S. 247). Genealogische Kritiken im besten Sinne erweisen sich als „Geschichten der Genese von Selbstverständnissen und der Konstruktion von Selbstverständnissen, adressiert an die Träger dieser Selbstbilder, und zwar in der Form von Machtgeschichten, die in den Zuhörern Zweifel an der Notwendigkeit ihres eigenen So-Seins auslösen oder verstärken“ (Saar 2007, S. 309).

Von Nietzsche und Foucault ausgehend, lässt sich eine kritische Organisationsforschung als genealogische Kritik im Foucaultschen Projekt einer „kritische[n] Ontologie der Gegenwart“ (Foucault 1992, S. 48) verorten, welche sich als „kritische Ontologie der modernen Organisationen“ (Hartz/Rätzer 2013, S. 11, Herv.i.O.) fortschreiben lässt. Das Ausgangsmotiv einer so verstandenen kritischen Organisationsforschung wäre dann, „die Geschichte der Gegenwart zu schreiben“ (Foucault 1977, S. 43), jedoch im Hinblick auf die spezifische und hier als konstitutiv verstandene Rolle, welche Organisationen in dieser Geschichte spielt bzw. spielen. Dabei wird von einer Ko-Evolution von Organisation und Gesellschaft ausgegangen und die Suche und die „Frage nach dem überzeitlichen ,Wesen“ von Organisation“ (Neuberger 1997, S. 494) problematisiert. Ein in dieser Hinsicht radikal historisierender Blick auf Organisationen ist zunächst eine notwendige Bedingung für jene von Foucault als philosophische Haltung verstandene „permanente Kritik unseres geschichtlichen Seins“ (Foucault 2005b, S. 699). Notwendig insofern, weil die Rekonstruktion der Gewordenheit von 
Organisationen als Rekonstruktion der Bedingungen der Möglichkeit von Organisationen diese zugleich in ihrer historischen Kontingenz im Sinne eines auch-anders-möglich-seiend erhellt. Im Kontext der CMS wird dieser Anspruch vielfach als Denaturalisierung formuliert (vgl. Fournier/Grey 2000; Alvesson et al. 2009). Denaturalisierung ist kein privilegierter Ansatz Foucaults oder der CMS, sondern ein wiederkehrendes Motiv kritischer Forschung schon Karl Marx sprach davon, dass man die „versteinerten Verhältnisse“ zum Tanzen zwingen müsse (1957, S. 381, vgl. auch Thompson/McHugh 2009, S. 13-14). Analog zu Foucaults Kritikverständnis ermöglicht eine solche Historisierung „Differenzen zu denken, für selbstverständlich Gehaltenes zu irritieren und in diesem Sinne Räume für Kritik zu öffnen“ (Bruch/Türk 2005, S. 120). Jenseits dieser Irritation erhält der radikal historisierende Blick im Kontext einer kritischen Organisationsforschung eine spezifische Zuspitzung, welche Organisation nicht nur als historisch gewordenen (und damit wie auch immer veränderbaren) sozialen Ort begreift, sondern diesen auch ,als eine spezifische Regierungsform der Moderne“ (Bruch/Türk 2005, S. 120) thematisiert, wie es etwa Ted Nace (2005) in seiner materialreichen Studie „Gangs of America“ instruktiv vorführt. Damit wird auch das für eine genealogische Kritik ,konstitutive Verhältnis zwischen Subjektivität und Macht“ (Saar 2009, S. 251) ausgeleuchtet. In der Perspektive Foucaults erscheinen Krankenhäuser, psychiatrische Anstalten, Gefängnisse, Kasernen, Fabriken, Manufakturen, Schulen nicht als mehr oder weniger vorausgesetzter, diskurs- und disziplinenkonstituierender Gegenstandsbereich, sondern als Resultat historischer Formen der Problematisierung des sozialen Geschehens und den damit verbundenen Lösungsversuchen einer Regierung des Sozialen. Dabei ist wichtig, dass Organisationen an-sich in dieser Perspektive weder einfach gut noch böse, weder einfach repressiv noch Vehikel der Befreiung sind (Benjamin 1972; Adorno 1953; Foucault 2005c, S. 331-332), wenngleich der genealogische Blick im Grundsatz machtkritisch verfährt. In der Figur der Problematisierung eröffnet sich schließlich ein direkter Bezug zur Thematik der Krise. Die Genealogie ermöglicht damit auch die historische Rekonstruktion von Krisen als Krisen des Organisierens und der Bemühungen um deren Überwindung.

Diese Perspektive der Ko-Evolution von Organisationen und Gesellschaft lässt sich durch einen Wechsel auf die Ebene der konkreten organisationalen Praktiken vertiefen. Die allägliche Aufrechterhaltung von Organisationen, sprich der Prozess des Organisierens, gerät in den Blick. Damit einher geht zunächst ein Perspektivenwechsel, welcher „Organisation nicht als strukturalistisch-abstraktes System“ betrachtet, sondern „,sich für den faktischen Konstitutions- und Reproduktionsprozess derjenigen sozialen Kontexte [interessiert], die die 
Alltagsakteure als >Organisation` bezeichnen“" (Türk 1993, S. 41). Dies verweist auf das Foucaultsche Interesse für eine Analyse der „Mikrophysik der Macht“ und eine ,politische Anatomie des Details“ (Foucault 1977, S. 178), schließt aber auch an das grundsätzlich prozesshafte Verständnis von Organisation in postmodernen und poststrukturalistischen Ansätzen der Organisationsforschung an (Chia 1995; Kneer 2008). Der Blick, welcher sich machtkritisch auf die alltäglichen „Kleinigkeiten und Kleinlichkeiten“ (Foucault 1977, S. 181) in Organisationen richtet, erlaubt die konkrete Erfassung der Genealogie und Transformation organisationsförmiger Praktiken und Verfahren und den damit verbundenen Wissensordnungen und Subjektivierungsweisen.

Der genealogische und mikrophysikalische Blick rekonstruiert dann Organisation nicht ,als einen neutralen, technischen Modus der effizienten Koordination von Ko-Operation“" sondern ermöglicht deren Dechiffrierung ,als typische Form von Herrschaft“ (Türk 1995, S. 41). An diesem Punkt trifft sich die genealogische Kritik mit der Perspektive der Arbeitsprozesstheorie (Braverman 1998 [1973]; Friedman 1977; Edwards 1979; Burawoy 1979) und deren herrschafts- und kontrolltheoretischen Lesart der modernen Organisation und erweist sich auch an die Arbeiten der „Radicals“ um Samuel Bowles, Herbert Gintis oder Stephen A. Marglin anschlussfähig (Bruch 1997). Aus genealogischer Perspektive sind dann „,Managementく und >Organisationく keine bloßen Objekte oder gegebene Einheiten, die man unvermittelt beobachten, beschreiben, vermessen oder klassifizieren könnte“, sondern „Produkte oder Effekte von Diskursen und Praktiken, die bestimmen, was gesehen wird und was nicht gesehen wird, was sichtbar und sagbar wird und zugleich, was unsichtbar und unsagbar wird“ (Weiskopf 2003, S. 14).

Zusammenfassend ermöglicht die Rekonstruktion von Organisationen als typischer Form von Herrschaft, einerseits Leiden in und durch Organisationen beredt werden zu lassen und dieses an die historisch sich wandelnden Formen des Organisierens zurückzubinden. Zudem läuft eine Organisationsforschung in genealogischer Absicht implizit in jenem emphatischen Fluchtpunkt zusammen, welchen man mit Foucault (1992, S. 15) als „Entunterwerfung“ bezeichnen kann. Wissenschaftliche Arbeiten in diesem Verständnis sind immer auch politische Projekte, gerichtet ,gegen den Gedanken universeller Notwendigkeiten im menschlichen Dasein. Sie helfen entdecken, wie willkürlich Institutionen sind, welche Freiheit wir immer noch haben und wie viel Wandel immer noch möglich ist" (Foucault 2005d, S. 961). Zugleich verbleibt genealogische Kritik oftmals in den Grenzen von Denaturalisierung und Herrschaftskritik. So mag sich die Einsicht einstellen, dass 
Organisation „nicht mehr so gesehen werden kann wie zuvor“ und die Geschichte des Organisierens keine emanzipatorische und lineare Geschichte der fortgesetzten Befreiung von (Arbeits-)Leid, Mangel und Not ist. Die Frage aber, wie es denn anders sein könnte, wird jedoch von der genealogischen Kritik nicht beantwortet. An dieser Frage orientiert sich der Vorschlag eines dritten Einsatzpunktes kritischer Organisationsforschung, welcher im Folgenden als welterschließende Kritik diskutiert wird.

\subsection{Kritische Organisationsforschung als welterschließende Kritik}

„If utopia is nowhere, it is not dangerous, but if it is somewhere, it is potentially very dangerous indeed. Particularly to those people who would rather that it stayed in books. " (Parker 2002b, S. 224)

„Fenster zu möglichen Welten zu öffnen, das jedenfalls bleibt die Aufgabe. “(Ortmann 2008, S. 23)

Welterschließende Kritik widmet sich nicht primär der Kritik organisationaler Rationalität oder Organisationen als Regierungsform der Moderne. Sie speist sich aus einer Affinität für Formen des Heterotopischen, anderen Orten, welche einerseits mit Gaston Bachelard (1987) „Bilder des glücklichen Raumes“ und andererseits mit Ernst Bloch (organisationale) Möglichkeiten des ,aufrechten Ganges“ entwerfen und zu realisieren versuchen. Kritische Organisationsforschung als welterschließende Kritik hätte zugleich den Anspruch, Befürchtungen entgegenzuarbeiten, dass der Raum des spezifisch Heterotopischen selbst sich zusammenzieht und die Gegenwart wesentlich durch die Metaphern einer „utopische[n] Unterernährung“ und einer „Impotenz im Antizipatorischen“ (Bloch 1975, S. 24) zu beschreiben wäre. Sie steht im Kontrast zur letztendlichen Düsternis der genealogischen Kritik und möchte einen Weg aus der Gebundenheit der Kritik an die jeweiligen organisationalen (Macht-)Verhältnisse weisen, welche zum einen die Gefahr der Resignation und Handlungsunfähigkeit in sich birgt und zum anderen die Gefahr eines romantisierenden Verlangens nach totaler Andersheit (Raffnsøe et al. 2016, S. 287-89) und die „Neigung, nach billigen, archaischen oder fantasierten Formen von Glück zu suchen“ (Foucault 2005c, S. 333) mit sich führt. In diesem Sinne wäre eine kritische Organisationsforschung als welterschließende Kritik eine organisationstheoretisch gewendete systematische Beschreibung ,die es sich zur Aufgabe machte, in einer bestimmten Gesellschaft diese andersartigen Räume, diese anderen Orte, diesen zugleich mythischen und realen Gegensatz 
zu dem Raum, in dem wir leben, zu erforschen, zu analysieren, zu beschreiben und zu >lesen`“ (Foucault 2005e, S. 936).

Für Foucault stehen Heterotopien in einer Funktionsbeziehung zum übrigen Raum, welche sich zwischen zwei Extrempolen bewegt: Heterotopien können als illusionärer Raum fungieren, wie in den Gärten des Orients, oder als ein anderer Raum der vollkommenen Ordnung, welcher sich, wie etwa in einzelnen Kolonien des 18. Jahrhunderts, gegen die wirre Unordnung des Realen stellt. Hinzu treten die sogenannten „Abweichungsheterotopien“, als „Orte, an denen man Menschen unterbringt, deren Verhalten vom Durchschnitt oder von der geforderten Norm abweicht“ (Foucault 2005e, S. 937). Somit steht in der Foucaultschen Skizze einer sogenannten Heterotopologie stärker das funktionale Element von Heterotopien - als Raum der Illusion, der Kompensation und der Abweichung - für den gesellschaftlichen Zusammenhang in den Vordergrund. In schillerndem Kontrast hierzu und nicht frei von Sozialkitsch - auch wenn man Foucault die fehlende Antizipation einer TV-Serie wie Traumschiff und des Booms der Kreuzfahrtindustrie nicht vorwerfen kann - schließt Foucault seinen Text emphatisch mit den Worten: „Das Schiff ist die Heterotopie par excellence. In den Zivilisationen, die keine Schiffe haben, versiegen die Träume. An die Stelle des Abenteuers tritt dort die Bespitzelung und an die Stelle der Freibeuter die Polizei“" (Foucault 2005e, S. 942, Herv. i.O.).

Hieran etwas nüchterner anknüpfend, ist es die Aufgabe welterschließender Kritik, den Blick auf organisationale Heterotopien zu richten, welche sich als andere Räume, als Gegenräume verstehen und sich in dieser Hinsicht rekonstruieren lassen. Heterotopien, welche oftmals entstanden sind als Reaktion auf die Krisenhaftigkeit des sonstigen gesellschaftlichen Raumes oder auf dessen wohlformulierter und durchgesetzter Wissens-, Macht- und Subjektordnung. Eine Auseinandersetzung mit Heterotopien kann dann auch aus der von Foucault thematisierten Sackgasse der Analytik der Macht führen, jener Sackgasse der Resignation, „,in die uns die Macht selbst führt, in unserem Leben wie in unserem Denken“ (Deleuze 1997, S. 133). Foucault hat den Weg aus dieser Sackgasse in den „Technologien des Selbst“" gesucht; in der (Selbst-)Reflexion als Dopplung des und Abkopplung von einem Außen „wird [es] stets eine Beziehung zu sich geben, die den Codes und den Mächten Widerstand leistet" (Deleuze 1997, S. 145). Hier soll hingegen vorgeschlagen werden, diesen Ausweg durch die Hinwendung zu anderen Räumen des Organisierens zu finden, als in diesem Sinne kritischen Heterotopien. Welterschließende Kritik nimmt dabei die Bewegung der immanenten Kritik in sich auf, insofern diese nun nach den möglichen Realisationen gesellschaftlicher Werte wie 
Freiheit, Gerechtigkeit oder Solidarität in realen und möglichen anderen Räumen fragt, als auch die Bewegung der genealogischen Kritik, indem diese ebenso dem Impuls folgt, dass Organisation immer auch anders sein kann. Welterschließende Kritik besitzt jedoch ein grundsätzlich affirmatives Moment, sie versteht sich als Antidot zu einer die kritische Theorie oftmals begleitenden Hermeneutik des Verdachts. Affirmativ meint dabei nicht naiv, sie folgt jedoch der Überzeugung, dass auch Organisationen vom Standpunkt ihrer „utopian possibilities“ (Parker et al. 2007, x) betrachtet werden können. Im Sinne von Alvesson und Deetz (2000, S. 151-153; Alvesson/Ashcraft 2009) kann Welterschließung schließlich zu einer „transformative re-definition“ des Wissens und der Praktiken des Organisierens beitragen, insofern diese die Imagination organisationaler Alternativen unterstützt. Im Folgenden sollen nun zwei mögliche Suchrichtungen welterschließender Kritik skizziert werden.

\subsubsection{Arbeit im Gelände - Rekonstruktion der Pluralität ökonomischer und organisationaler Formen}

„Ich bin ... ein Kartograph“ (Foucault 2005f, S. 895)

War der Foucault der genealogischen Kritik der Kartograph der Disziplinen und der Einschließung, muss die welterschließende Kritik sich einer Kartographie des anderen Organisierens zuwenden. Einfache Fragen können hier am Anfang stehen: Was haben wir im Blick, wenn wir an Organisationen und Unternehmen denken? Welche Fallstudien diskutieren wir in der Lehre? Welche Pluralität lassen wir im Denken über Organisationen, Märkte und Ökonomien zu? Was könnte eine gute Organisation auszeichnen? Welterschließende Kritik setzt zunächst an der Wahrnehmung und Sichtbarmachung von Alternativen an. Für Zanoni und KollegInnen (2017) zielt dies auf eine Erweiterung des Archivs des organisational Vorstellbaren. Diese Archivierung im Sinne Derridas ,always performs specific realities in the present that are working to bring about a specific future“ (Zanoni et al. 2017, S. 580). Das Motiv von Parker und KollegInnen, ein Dictionary of Alternatives vorzulegen, entspringt entsprechend dem Eindruck das ,[o]ne of the most common pieces of common sense nowadays is that there is no real alternative to market managerialism“ (2007, ix). Damit folgen sie Fredric Jameson (1994, xii), welcher konstatiert: „It seems easier for us today to imagine the thoroughgoing deterioration of the earth and of nature than the breakdown of late capitalism; perhaps that is due to some weakness in our imaginations“. Das Routledge 
Companion to Alternative Organization (Parker et al. 2014a) setzt sich zum Ziel ,to move beyond complaining about the present and into exploring this diversity of organizational possibilities“ (ebd., Vorwort). Entlang der Gegenstandsbereiche Arbeit (hier finden sich bspw. Beiträge über mitarbeitergeführte Unternehmen, Kommunen, Familienarbeit) Austausch und Konsum (bspw. Fair Trade, lokale Währungen, Gabentausch, Ehrenamt, solidarische Landwirtschaft) sowie Ressourcen (bspw. Commoning, Ökobilanzen, Open Source) wird hierbei die Vielfalt alternativer Organisationsformen aufgeblendet und auch kritisch diskutiert. Für eine Diskussion alternativer Organisationsformen schlagen Parker und Kolleginnen eine Orientierung an den Werten der individuellen Autonomie, der Solidarität und Nachhaltigkeit vor. Als Orientierungen oder Leitplanken weisen diese Prinzipien wiederum darauf hin, dass die Bewertung von heterotopen Organisationen hinsichtlich ihres alternativen Charakters nicht a priori vorgenommen werden kann: ,[I]t simply isn't possible to say that there are some arrangements that are unambigiously good, and others that are unambigiously bad“" (Parker et al. 2014b, S. 31). Es ist schließlich das Verdienst der Arbeiten von Gibson-Graham (2006a, 2006b, 2008; Zanoni 2017) mit der Einführung der Perspektive der „diverse economies“ die Frage der alternativen Ökonomien und Organisationen weiter systematisiert zu haben. Gibson-Graham verstehen ihre Arbeit als ein „performative ontological project“" (Gibson-Graham 2008, S. 1), welches den monologischen und hegemonialen Erzählungen über den Markt, den Kapitalismus oder die Organisation zunächst den Aufweis der Pluralität von im herrschenden Diskurs meist unsichtbaren oder marginalisierten ökonomischen Aktivitäten entgegensetzt. Metaphorisch lässt sich bezogen auf die Vielfalt ökonomischer Praktiken von einem Eisberg sprechen, wobei eine Vielzahl ökonomischer und organisationaler Aktivitäten unter der Wasseroberfläche stattfinden, sprich unterhalb der Schwelle der signifikanten Wahrnehmung des akademischen und öffentlichen Diskurses. Gibson-Graham unterscheiden im Sinne ihrer Kartierung dabei drei ökonomische Praktiken: Transaktionen, Arbeit, Unternehmensformen und weisen auf die Diversität jenseits der dominanten Vorstellungen von Markt, Lohnarbeit und kapitalistischer Unternehmung hin. Hierunter fallen diverse Formen alternativ-marktlicher (bspw. Schwarzmärkte, informelle Märkte, Tauschhandel) und nicht-marktlicher Transaktionen (bspw. in Privathaushalten, beim Gabentausch, bei staatlicher Verteilungspolitik), plurale Formen von Arbeit (bspw. Kooperativen, Haushaltsarbeit, Ehrenamt) und schließlich von Unternehmen (bspw. staatliche Unternehmen, Non-Profit Organisationen, kommunale Unternehmen). Hierin anknüpfend wäre es zunächst die Aufgabe einer kritischen Organisationsforschung an dieser Sichtbarmachung von Vielfalt mitzuwirken, die Pluralität des Organisierens zu kartieren, 
mögliche (eigene) Vorurteile hinsichtlich der Marginalität und des Utopismus anderer und alternativer Formen des Arbeitens und Organisierens zu reflektieren sowie die vorhandene Pluralität in den wissenschaftlichen Diskurs als auch in die akademische Lehre (Reedy/Learmonth 2009) einzuspeisen.

Neben dieser zunächst affirmativen Perspektive, bedarf es jedoch zweitens einer erweiterten Reflexion alternativen Organisierens vor dem Hintergrund des Topos von Organisation als Regierungsform der Moderne. Zunächst lassen sich auch hier eine Reihe von Fragen stellen: Inwieweit finden sich in der Pluralität alternativer Entwürfe emanzipatorische Perspektiven, welche als Politiken der Entunterwerfung lesbar sind? Welche organisationalen Mechanismen, welche gesellschaftlichen Kontexte und Rahmenbedingungen befördern, restringieren oder führen zum Scheitern emanzipatorischer Praktiken (Barratt 2008, S. 523528)? Welche Reichweite, welche zeitliche Perspektive, welche Übertragbarkeit besitzen jene alternativen Entwürfe (Parker et al. 2014c, S. 362f.)? Welche Impulse können alternative Praktiken für den Mainstream des Managens und Organisierens setzen (Hartmann 2014, S. 623-624), welche Formen der Kooptation und der herrschaftsstabilisierenden Einhegung lassen sich feststellen (Boltanski und Chiapello 2006)? So weist schließlich Žižek kritisch darauf hin, dass eine reine Erfindung oder Vorstellung ,anderer Welten" zumindest zwiespältig ist: „Alles hängt davon $a b$, in welcher Beziehung diese Fiktionen zum zugrundeliegenden Realen des Kapitalismus stehen - ergänzen sie diesen nur durch imaginative Vielfalt, wie die postmodernen >lokalen Erzählungen`, oder stören sie sein Funktionieren?“`(Žižek 2009, S. 95, Herv.i.O.).

Daneben sollte eine welterschließende Kritik zwei weitere reflexive Momente in die Betrachtung alternativer Organisationsformen einführen. Zunächst kann sie aus der Perspektive der Organisationsforschung auf die Gefahren der Schließung und der bündischen Funktion, wie diese bereits bei Max Weber thematisiert wurde, auf die Gefahr der Machtkonzentration als auch der potentiell wiederum leidvollen (Selbst-)Ausbeutung hinweisen und diese in den heterotopen Diskurs einspeisen. Hier erweist sich ein genealogischer Blick auf historische Versuche alternativen Organisierens als lehrreich, in welchen etwa Phänomene des Konformismus, der mangelnden Partizipation aufgrund zeitlicher Restriktionen oder als defizitär empfundene kommunikative Fähigkeiten sowie mangelnde ökonomische Ressourcen immer wieder zum Scheitern alternativer Projekte führte (Barratt 2008, S. 525-528; Young 1977). Zum zweiten wäre es die Aufgabe, im Bereich der Heterotopien notwendige Differenzierungen vorzunehmen, welche es erlauben, kritische 
Heterotopien von Formen der organisationsförmigen Einhegung unter Verwertungsgesichtspunkten, wie es etwa im Bereich der Sharing Economy zu beobachten ist, zu unterscheiden. Insofern ist eine welterschließende Kritik zunächst affirmativ, da diese offen ist für die „Intensitäten“ (Lyotard 1978) und Energien kritischer Heterotopien. Ein zynisches Bewusstsein ist ihr fremd. Zugleich sollte sich eine welterschließende Kritik immer der Fallstricke und Gefahren des Organisierens und der Rolle von Organisationen im gesellschaftlichen Kontext und dessen Transformationen bewusst sein, welches wiederum auf die Einsatzpunkte immanenter und genealogischer Kritik zurückweist.

\subsubsection{Jenseits des „Manufacturing Consent“" - Innerorganisationale Heterotopien als Vorschein alternativer Arbeits- und Organisationsformen}

Eine zweite Suchrichtung welterschließender Kritik folgt der Idee, dass sich untersuchenswerte heterotope Momente und Wirklichkeitsschichten sowohl im sprachlichdiskursiven Raum als auch im Bereich der Taktiken und Verhaltensweisen im (organisationalen) Alltag selbst auffinden lassen. Der sprachlich-diskursive Raum lässt sich mit einem Begriff Henri Levebres als „Repräsentationsraum“ bezeichnen. „Repräsentationsräume“ sind - in Abgrenzung zu den „Raumrepräsentationen“, welche die Produktionsverhältnisse, die gesellschaftliche Ordnung repräsentieren - der ,gelebte Raum, vermittelt durch die Bilder und Symbole, die ihn begleiten, also ein Raum der >Bewohner`, der >Benutzer $<[. .$.$] . Es ist der beherrschte, also erlittene Raum, den die Einbildungskraft zu$ verändern und sich anzueignen versucht. Er legt sich über den physischen Raum und benutzt seine Objekte symbolisch [...]“ (Levebre 2006, S. 336). Hinsichtlich heterotoper Taktiken und Verhaltensweisen in Organisationen lässt sich weiter an Michel de Certeau anschließen, der ergänzend zur Foucaultschen Analytik der Disziplinarmechanismen fragt: „Aber welche Umgangsweisen mit dem Raum entsprechen diesen einen disziplinären Raum erzeugenden Apparaten, wenn man [mit der] Disziplin spielt?“ (De Certeau 1988, S. 187; vgl. auch Hjorth 2005; Ortmann/Sydow 2017). Mit anderen Worten geht es hierbei um die oftmals „geräuschlosen“, „stillen Produktionen“ der (Wieder-)Aneignung und Umformung des beherrschten und organisierten Raumes. Anknüpfen lässt sich auch an eine lange Traditionslinie der Arbeits- und Industriesoziologie sowie der Organisationsforschung, welche sich immer wieder mit dem Wechselverhältnis von organisationaler Macht und Formen des Widerstands und der Subversion auseinandergesetzt hat. Prominent stehen hierfür Beiträge der Arbeitsprozesstheorie, welche Fragen der Kontrolle des Arbeitsprozesses etwa 
unter der Perspektive einer Dialektik von Kontrolle und Widerstand (Edwards 1979), des widerspruchsvollen Verhältnisses von direkter Kontrolle und verantwortlicher Autonomie (Friedman 1977) oder eines „Manufacturing Consent“ (Burawoy 1979) diskutiert haben. Im Sinne der Tragfähigkeit dieser Suchrichtung ist dabei eine weiterführende Auseinandersetzung mit der vielfältig formulierten organisationstheoretischen Diagnose der letztlich funktionalen, d.h. sowohl produktiven als auch machtstabilisierenden Einbindung von Widerstand, Subversion und Versuchen der (Wieder-)Aneignung notwendig (vgl. exemplarisch Burawoy 1979; Fleming/Spicer 2003; Contu 2008; Al-Ani 2013). Dass diese Einhegung vielfältig stattfindet, ist unbestritten, gleichwohl ist es notwendig, im Sinne einer welterschließenden Kritik bei dieser Diagnose nicht stehenzubleiben. Einen heuristischen Einsatzpunkt bieten dabei wiederum die Arbeiten von Deleuze und Foucault. In der Deleuzschen Diskussion des Dispositivkonzeptes, als spezifische Relation von Wissens-, Macht- und Subjektivierungslinien, liegt eine Möglichkeit, Differenzen und Widerständigkeiten gegenüber den tradierten Formen und Vorstellungen von Organisation in den Blick zu bekommen. Für Deleuze sind es emphatisch die Subjektivierungslinien, welche die Schließung eines Dispositivs verhindern können, welche sich „den Dimensionen des Wissens und der Macht entziehen“ (Deleuze 1991, S. 159), (organisationale) Dispositive transformieren und das Kreative, das Neuartige in die Welt setzen (Hartz 2017). Für Foucault ist es im Kontext der Diskussion des Pastorats und des Begriffs der Führung der wiederholte Rekurs auf ein „Gegen-Verhalten“, auf „Verhaltensrevolten“, wobei „eine unmittelbare und grundlegende Korrelation zwischen der Verhaltensführung und dem Gegen-Verhalten“ (Foucault 2004, S. 284) vorliegt. Dies schließt an die vielzitierte Formel an: „Wo es Macht gibt, gibt es Widerstand“ (Foucault 1983, S. 116). Im Verhältnis zur Macht „gibt es ... nicht den einen Ort der Großen Weigerung [...]. Sondern es gibt einzelne Widerstände: mögliche, notwendige, unwahrscheinliche, spontane, wilde, einsame, abgestimmte, kriecherische, gewalttätige, unversöhnliche, kompromissbereite, interessierte oder opferbereite Widerstände, die nur im strategischen Feld der Machtbeziehungen existieren können“" (Foucault 1983, S. 117). Für die hier diskutierte Perspektive ist dabei wichtig festzuhalten, dass Widerstand nicht bloß die Negativform oder die Folge von Macht ist und insofern, wie auch Foucault festhält, nicht nur „trügerische Hoffnung und notwendig gebrochenes Versprechen“ ist (Foucault 1983, S. 117). In anderer Diktion und direkt am Gegenstand der Organisation orientiert, wäre hier auch die an Cornelius Castoriadis geschulte Konzeptualisierung des Verhältnisses von organisationaler Macht und Widerstand durch Harald Wolf anzuführen, für welchen die Idee der Autonomie als Gegenentwurf zur organisationalen Heteronomie sich nicht stillstellen oder 
befrieden lässt. Dieser Entwurf der Autonomie ,artikuliert sich auch in der Produktionssphäre. Es gibt in ihr einen imaginären Gegenstrom, aus dem sich der Sinn einer autonomen Institution der Arbeit herauslesen lässt" (Wolf 1999, S. 131). Dabei ist auch für Wolf klar, dass dieser „Gegenstrom ... in ... gesellschaftlichen Kompromissbildungen ... mündet" und den radikalen Sinn der autonomen Arbeit vor allem die „Seitenarme und Unterströmungen“ der Gesellschaft mit sich führen, welche ,immer wieder zu versiegen und verdrängt zu werden drohen“" (ebd.). Gleichwohl gilt es hier programmatisch und dann auch empirisch anzuschließen, denn „die Forderungen [nach Autonomie] sind da, und sie machen sich im Denken und Handeln immer wieder geltend - selbst an Orten, an denen man es nicht vermutete, und in Formen, denen man es auf den ersten Blick nicht ansah" (ebd., S. 135). Ganz ähnliche Überlegungen finden sich auch in Günther Ortmanns Untersuchung über Personalinformationssysteme als „Architektur der Disziplin“: „In Entwürfen künstlicher, lebloser Ordnungen trifft sich solche >Theorie als design` mit den Techniken der Disziplin, wie Foucault sie beschrieben hat - und wieder verraten Spuren der Angst vor chaotischer Lebendigkeit das Motiv eines auffälligen, drängenden Gestaltungswillens“ (Ortmann 1984, S. 10f.). Hieran anschließend sollte sich eine welterschließende Kritik als ein Gegengift gegenüber der auch berechtigten skeptischen Rahmung von Widerstand oder der vermeintlich abgeklärten Rede von „Schaukämpfen“ und Widerständen als Verstärkung des „Immunsystems“ zum Zwecke des Systemerhalts (Al-Ani 2013, S. 148) erweisen. Für Peter Fleming und André Spicer erweisen sich in dieser Hinsicht Praktiken des Widerstandes und der Subversion als in mehrfacher Hinsicht mit Fragen der Gerechtigkeit verknüpft: ,[A]lmost all these flash points of struggle seem to point to some elemental concern for a fair distribution of resources, the recognition of oneself as a dignified and meaningful person, and the right to make decisions within the organization“" (Fleming/Spicer 2007, S. 150). Dies führt auch zurück zu den diskutierten Ausgangspunkten von Kritik, seien es Erfahrungen des Leidens, hier substantiierbar als ökonomische Ausbeutung und Deprivation oder Missachtungs- und Exklusionserfahrungen im organisationalen Kontext, seien es krisenhafte gesellschaftliche Entwicklungen oder gegenwärtige soziale Kämpfe. Damit zeigt sich auch an, dass die Suche nach innerorganisationalen Heterotopien oftmals wiederum den Blick über die Organisationsgrenzen hinaus lenkt und damit rückbindet an die gesellschaftliche Einbettung von Organisationen und die diskutierte Ko-Evolution von Organisation und Gesellschaft.

Insofern Organisation eine Form der Vergesellschaftung, „ein von Menschen für Menschen geschaffenes ist“" (Adorno 1953, S. 447), begreift welterschließende Kritik, als Modus der Reflexion über andere und alternative Formen des Organisierens, Organisation nicht an sich 
als Drohung. Vielmehr beharrt sich darauf, dass die Zwecke von Organisationen „menschliche Zwecke und grundsätzlich von Menschen zu verändern [sind], so schwer nachvollziehbar den meisten heute auch die Möglichkeit sein mag“ (ebd., S. 446).

\section{Résumé}

Das Ziel des vorliegenden Essays war es, die Perspektive kritischer Organisationsforschung $\mathrm{zu}$ konturieren und insbesondere entlang unterschiedlicher Einsatzpunkte von Kritik eine Möglichkeit der Strukturierung des Feldes vorzuschlagen. Kritik, so der Ausgangspunkt der Betrachtung, befindet sich auf notorisch schwankendem Boden und auch deren aktueller Zustand stimmt alles andere als optimistisch. Hier im trüben Fahrwasser Orientierungspunkte zu setzen (und dieses Fahrwasser zugleich ein wenig aufzuhellen), erschien somit geboten und soll nun abschließend nochmals zusammenführend diskutiert werden.

Diese Orientierung erfolgte zunächst durch eine Rückbesinnung auf drei Motive kritischer Praxis: Leiden, Krise und Notwendigkeiten der Gegenwart. Die Rekurse auf Leiden und Krise erweisen sich dabei historisch als immer wieder mächtige Impulse der Artikulation von Kritik. Beide Ausgangspunkte sind gleichwohl nicht trivial. Leid und dessen Artikulation sind nicht nur Gegenstand diskursiver Kämpfe und Deutungen, sondern laufen Gefahr, sich in Reaktionen reiner Empörung und oder des lustvollen Hinstarrens zu erschöpfen. Krisen erweisen sich wiederum als hochgradig zeitbezogen und erfahren in ökonomischen und organisationalen Kontexten vielfach positiv konnotierte Umdeutungen. Das herausgestellte Motiv der „Notwendigkeiten der Gegenwart“ erweist sich insofern als offener und weiter gefasst, weil es sich nicht notwendig auf Leiden und Krisen bezieht, sondern stärker und offensiver die Frage der Veränderung adressieren kann.

Hieran anschließend, wurden mit immanenter, genealogischer und welterschließender Kritik drei mögliche Einsatzpunkte kritischer Organisationsforschung diskutiert. Die klassische immanente Kritik orientiert sich dabei an der Kluft von Anspruch und Wirklichkeit in Organisationen und erweist sich mit Blick auf die Geschichte der Organisationsforschung oftmals als Rationalitätskritik oder als Kritik an den „dunklen Seiten“ des Organisierens und des Managements. Sowohl der Bezug auf organisationale Krisenanfälligkeit und -haftigkeit als auch - wie in der Perspektive der „dark sides“ - auf intendiertes und nicht-intendiertes Leiden in und an Organisationen konturieren die exemplarisch angeführten Perspektiven und Studien als einen ersten Einsatzpunkt kritischer Organisationsforschung. Die genealogische 
Kritik stützt sich hingegen primär auf die Perspektive der Foucaultschen Genealogie und Geschichte der Gegenwart. Genealogische Kritik als Machtkritik interessiert sich einerseits für eine Rekonstruktion von Organisation als Regierungsform der Moderne, weißt andererseits jedoch auf die historische Kontingenz der modernen Organisationen hin. Auch hier ist der Rekurs auf Leiden - in der Zusammenführung von Macht- und Subjektivierungskritik - und Krisen, welche als historische Formen der Problematisierung von Organisation erscheinen, evident. Mit dem Aufweis der historischen Kontingenz von Organisation öffnet sich darüber hinaus ein Fenster, welches den systematischen Ausgangspunkt der hier vorgeschlagenen welterschließenden Kritik als dritten Einsatzpunkt kritischer Organisationsforschung bildet. Der Einsatz der welterschließenden Kritik besteht darin, im Aufzeigen von Formen ,anderen Organisierens“ und innerorganisationalen Heterotopien einen dritten Weg zwischen Affirmation und Kritik zu beschreiten. Unter Bezug auf das Konzept der Heterotopie geht es zunächst um eine aktive Suche nach und die Sichtbarmachung von alternativen Formen des Organisierens. Insofern ist welterschließende Kritik affirmativ, weil diese sich erstens in das „Drängeln der Gegenwart“ stellt und die dort vorhandenen Bemühungen um alternative Formen des Organisierens aufgreift und zweitens die Geschichte der modernen Organisation nach Alternativen jenseits einer Sicht auf Organisationen als Regierungsform der Moderne befragt. Dies schließt auch den Blick auf innerorganisationale Heterotopien ein. Diese Affirmation ist zugleich nicht naiv. Vielmehr oszilliert welterschließende Kritik zwischen Affirmation und Kritik, insofern diese mit dem Erbe der immanenten und genealogischen Kritik notwendige Reflexionsschleifen in das Konzept der Heterotopie einzieht. Welterschließende Kritik erweist sich dann als ein möglicher Weg aus den Trübnissen und Erschöpfungszuständen einer Permanenz der Kritik, ohne das reiche Erbe immanenter und genealogischer Kritik aufzugeben. 


\section{Literaturverzeichnis}

Adler, P. (2016): Alternative Economic Futures: A Research Agenda for Progressive Management Scholarship. In: The Academy of Management Perspectives 30 (2), S. 123-128.

Adler, P./Forbes, L./Willmott, H. (2007): Critical Management Studies. In: Academy of Management Annals 1, S. 119-179.

Adorno, T. W. (1953): Individuum und Organisation. In: Soziologische Schriften I. Frankfurt/Main: Suhrkamp, S. 440-456.

Adorno, T. W. (1954): Beitrag zur Ideologienlehre. In: Soziologische Schriften I. Frankfurt/Main: Suhrkamp, S. 457-477.

Adorno, T. W. (1966): Negative Dialektik. Frankfurt/Main: Suhrkamp.

Adorno, T. W. (1977): Kritik. In: Kulturkritik und Gesellschaft II. Frankfurt/Main: Suhrkamp, S. 185-793.

Adorno, T. W. (2010): Probleme der Moralphilosophie. Berlin: Suhrkamp.

Al-Ani, A. (2013): Widerstand in Organisationen. Organisationen im Widerstand. Virtuelle Plattformen, Edupunks und der nachfolgende Staat. Springer VS, Wiesbaden.

Alvesson, M./Bridgman, T./Willmott, H. (2009): Introduction. In: Alvesson, M./Bridgman, T./Willmott, H. (Hrsg.): The Oxford handbook of critical management studies. Oxford: Oxford University Press, S. 1-26.

Alvesson, M./Deetz, S. (2000): Doing critical management research. London: Sage.

Alvesson M, Ashcraft KL (2009) Critical Methodology in Management and Organization Research. In: Buchanan DA, Bryman A (Hrsg) The SAGE handbook of organizational research methods. Sage, Los Angeles, S. 61-77.

Bachelard, G. (1987): Poetik des Raumes. 8. Aufl. Frankfurt/Main: Fischer.

Barnard, Ch. I. (1968): The functions of the executive. Cambridge: Harvard University Press.

Barratt, E. (2008): The later Foucault in organization and management studies. In: Human Relations 61 (4), S. 515-537.

Benjamin, W. (1972): Ein Außenseiter macht sich bemerkbar. Zu S. Kracauer, »Die Angestellten «. In: Gesammelte Schriften: III: Kritiken und Rezensionen. Frankfurt/Main: Suhrkamp, S. 219-225.

Benson, J. K. (1983): Paradigm and praxis in organizational analysis. In: Research in Organizational Behaviour 5, S. 33-56.

Bloch, E. (1975): Hoffnung mit Trauerflor. In: Traub, R./Wiesner, H. (Hrsg.): Gespräche mit Ernst Bloch. Frankfurt/Main: Suhrkamp, S. 13-27.

Boltanski, L./Chiapello, È. (2006): Der neue Geist des Kapitalismus. Konstanz: UVK.

Bormann, K. v. (1973): Kritik. In: Krings, H./Baumgartner, H.M./Wild, Ch. (Hrsg.): Handbuch philosophischer Grundbegriffe, Bd. 3. 6 Bände. München: Kösel-Verlag, S. 807823.

Braverman, H. (1998 [1973]): Labor and monopoly capital. The degradation of work in the twentieth century. 25th anniversary. New York: Monthly Review Press.

Bruch, M. (1997): Betriebliche Organisationsform und gesellschaftliche Regulation. Zum Problem des Verhältnisses von Organisation und Gesellschaft in polit-ökonomisch 
orientierten Ansätzen. In: Ortmann G, Sydow J, Türk K (Hrsg) Theorien der Organisation. Die Rückkehr der Gesellschaft. Westdeutscher Verlag, Opladen, S 181-210.

Bruch, M./Türk, K. (2005): Organisation als Regierungsdispositiv der modernen Gesellschaft. In: Jäger, W./Schimank, U. (Hrsg.): Organisationsgesellschaft. Facetten und Perspektiven. Wiesbaden: VS Verlag, S. 89-123.

Brunsson, N. (1989): The organization of hypocrisy: talk, decisions and actions in organizations. London: Wiley.

Burawoy, M. (1979): Manufacturing consent. Changes in the labor process under monopoly capitalism. Chicago Ill. u.a.: Univ. of Chicago Press.

Butler, J. (1991): Das Unbehagen der Geschlechter. Frankfurt/Main: Suhrkamp.

Butler, J. (2009): Was ist Kritik? Ein Essay über Foucaults Tugend. In: Jaeggi, R./Wesche, T. (Hrsg.): Was ist Kritik? Frankfurt/Main: Suhrkamp, S. 221-246.

Celikates, R. (2009): Kritik als soziale Praxis. Gesellschaftliche Selbstverständigung und kritische Theorie. Frankfurt/Main, New York: Campus.

Certeau, M. d. (1988): Kunst des Handelns. Berlin: Merve Verlag.

Chia, R. (1995): From Modern to Postmodern Organizational Analysis. In: Organization Studies 16 (4), S. 579-604.

Cohen, M. D./March, J. G./Olsen, J. P. (1972): A garbage can model of organizational choice. In: Administrative Science Quarterly, 17 (1), S. 1-25.

Contu, A. (2008): Decaf resistance: On misbehavior, cynicism, and desire in liberal workplaces. In: Management Communication Quarterly 21 (3), S. 364-379.

Cooke, B. (2008): If Critical Management Studies Is Your Problem. In: Organization 15 (6), S. 912-914.

Cooper, R./Burrell, G. (1988). Modernism, Postmodernism and Organizational Analysis: An Introduction. Organization Studies 9 (1), 91-112.

Crouch, C. (2011): The strange non-death of neoliberalism. Cambridge: Polity Press.

Crozier, M./Friedberg, E. (1979): Macht und Organisation. Die Zwänge kollektiven Handelns. Königstein/Ts.: Athenäum.

Deleuze, G. (1991): Was ist ein Dispositiv? In: Ewald F, Waldenfels B (Hrsg) Spiele der Wahrheit. Michel Foucaults Denken. Suhrkamp, Frankfurt/Main, S 153-162.

Deleuze, G. (1997): Foucault. Frankfurt/Main: Suhrkamp.

Edwards, R. (1979): Contested terrain. The transformation of the workplace in the twentieth century. New York: Basic Books.

Fleming, P./Spicer, A. (2003): Working at a cynical distance: Implications for power, subjectivity and resistance. In: Organization 10 (1), S. 157-179.

Fleming, P./Spicer, A. (2007): Contesting the corporation. Struggle, power and resistance in organizations. Cambridge: Cambridge University Press.

Flyvbjerg, B. (2001): Making social science matter. Why social inquiry fails and how it can succeed again. Cambridge: Cambridge University Press.

Flyvbjerg, B. (2003): Making Organization Research Matter: Power, Values and Phronesis. In: Czarniawska, B./Sevón, G. (Hrsg.): The Northern Lights. Organization theory in Scandinavia. Malmö: Liber, S. 357-381. 
Foucault, M. (1975): Der Fall Rivière. Materialien z. Verhältnis von Psychiatrie u. Strafjustiz. Frankfurt/Main: Suhrkamp.

Foucault, M. (1977): Überwachen und Strafen. Die Geburt des Gefängnisses. Frankfurt/Main: Suhrkamp.

Foucault, M. (1992): Was ist Kritik? Berlin: Merve.

Foucault, M. (1983): Der Wille zum Wissen. Sexualität und Wahrheit 1. Suhrkamp, Frankfurt/Main.

Foucault, M. (1998): Über Hermaphrodismus. Der Fall Barbin. Frankfurt/Main: Suhrkamp.

Foucault, M. (2004): Die Geburt der Biopolitik. Geschichte der Gouvernementalität II. Frankfurt/Main: Suhrkamp.

Foucault, M. (2005a): Das Leben der infamen Menschen. In: Schriften in vier Bänden. Dits et Ecrits, Bd. 3. Frankfurt/Main: Suhrkamp, S. 309-332.

Foucault, M. (2005b): Was ist Aufklärung? In: Schriften in vier Bänden. Dits Et Ecrits, Bd. 4. Frankfurt/Main: Suhrkamp, S. 687-707.

Foucault, M. (2005c): Raum, Wissen und Macht. In: Schriften in vier Bänden. Dits et Ecrits, Bd. 4. Frankfurt/Main: Suhrkamp, S. 324-341.

Foucault, M. (2005d): Wahrheit, Macht, Selbst. In: Schriften in vier Bänden. Dits et Ecrits, Bd. 4. Frankfurt/Main: Suhrkamp, S. 959-966.

Foucault, M. (2005e): Von anderen Räumen. In: Schriften in vier Bänden. Dits et Ecrits, Bd. 4. Frankfurt/Main: Suhrkamp, S. 931-942.

Foucault, M. (2005f): Auf dem Präsentierteller. In: Schriften in vier Bänden. Dits et Ecrits, Bd. 2. Frankfurt/Main: Suhrkamp, S. 888-895.

Fournier, V./Grey, Ch. (2000): At the critical moment: Conditions and prospects for critical management studies. In: Human Relations 53 (1), S. 7-32.

Friedman, A. L. (1977): Industry and labour. Class struggle at work and monopoly capitalism. London: Macmillan.

Galtung, J. (1969): Violence, peace and peace research. In: Journal of Peace Research, 6 (3), S. 167-191.

Geuss, R. (1983): Die Idee einer kritischen Theorie. Königstein/Ts.: Hain.

Ghoshal, S. (2005): Bad Management Theories Are Destroying Good Management Practices. In: Academy of Management Learning and Education 4 (1), S. 75-91.

Gibson-Graham, J. K. (2006a): The end of capitalism (as we knew it). A feminist critique of political economy. Minneapolis, London: University of Minnesota Press.

Gibson-Graham, J. K. (2006b): A postcapitalist politics. Minneapolis, London: University of Minnesota Press.

Gibson-Graham, J. K. (2008): Diverse economies: performative practices for 'other worlds'. In: Progress in Human Geography 32 (5), S. 613-632.

Griffin, R. W./O'Leary-Kelly, A. (2004): The Dark Side of Organizational Behavior. San Francisco: John Wiley \& Sons, Inc.

Hartmann, R. K. (2014): Subversive functionalism: For a less canonical critique in critical management studies. In: Human Relations 67 (5), S. 611-632. 
Hartz, R. (2012): Reclaiming the Truth of the Market in Times of Crisis: Course, Transformation and Strategies of a Liberal Discourse in Germany. In: Culture and Organization, 18(2), S. 139-154.

Hartz, R. (2013): „Eine Art von ständigem ökonomischen Tribunal“ - Qualitative Befunde zur Semantik des Scheiterns in der diskursiven Bearbeitung der globalen Finanzkrise. In: Bergmann, J./Hahn, M./Langhof, A./Wagner, G. (Hrsg.): Scheitern - Organisations- und wirtschaftssoziologische Analysen. Wiesbaden: Springer VS, S. 301-321.

Hartz, R. (2017): Über die Kunst, sich anders zu organisieren - Organisation als „GegenDispositiv“" am Fallbeispiel einer Genossenschaftsbank. In: Diaz-Bone, R./Hartz, R. (Hrsg.): Dispositiv und Ökonomie. Diskurs- und dispositivanalytische Perspektiven auf Märkte und Organisationen. Wiesbaden: Springer VS, S. 203-231.

Hartz, R./Rätzer, M. (2013). Einführung. In: Hartz, R./Rätzer, M. (Hrsg.), Organisationsforschung nach Foucault. Macht - Diskurs - Widerstand (S. 7-15). Bielefeld: transcript.

Hassard, J./Parker, M. (Hrsg.). (1993). Postmodernism and organizations. London: Sage.

Hjorth, D. (2005): Organizational Entrepreneurship. With de Certeau on Creating Heterotopias (or Spaces for Play). In: Journal of Management Inquiry 14 (4), S. 386-398.

Horkheimer, M./Adorno, Th. W. (1988): Dialektik der Aufklärung. Philosophische Fragmente. Frankfurt/Main: Fischer Taschenbuch Verlag.

Jaeggi, R./Wesche, T. (2009): Einführung: Was ist Kritik? In: Jaeggi, R./Wesche, T. (Hrsg.): Was ist Kritik? Frankfurt/Main: Suhrkamp, S. 7-20.

Jäger, S. (2012): Kritische Diskursanalyse. Eine Einführung. 6. Aufl. Münster, Westf: Unrast. Jameson, F. (1994): The seeds of time. New York: Columbia University Press.

Kneer, G. (2008): Institution/Organisation: Über die Paradoxie des Organisierens. In: Moebius, S./Reckwitz, A. (Hrsg.): Poststrukturalistische Sozialwissenschaften. Frankfurt a.M: Suhrkamp, S. 124-140.

Koselleck, R. (2010): Begriffsgeschichten. Studien zur Semantik und Pragmatik der politischen und sozialen Sprache. Frankfurt/Main: Suhrkamp.

Levebre, H. (2006): Die Produktion des Raumes. [Auszug]. In: Dünne, J./Günzel, St./ Doetsch, H./Lüdeke, R. (Hrsg.): Raumtheorie. Grundlagentexte aus Philosophie und Kulturwissenschaften. Frankfurt/Main: Suhrkamp, S. 330-342.

Linstead, S. (2002). Organizational Kitsch. Organization 9 (4), 657-682.

Linstead, S./Maréchal, G./Griffin, R. W. (2014): Theorizing and researching the dark side of organization. In: Organization Studies 35 (2), S. 165-188.

Lyotard, J.-F. (1978): Intensitäten. Berlin: Merve.

Lyotard, J.-F. (1986). Das postmoderne Wissen. Ein Bericht. Wien: Passagen.

Marens, R. (2010): Speaking Platitudes to Power: Observing American Business Ethics in an Age of Declining Hegemony. In: Journal of Business Ethics 94, S. 239-253.

Marx, K. (1957): Zur Kritik der Hegelschen Rechtsphilosophie. Einleitung. In: Karl Marx und Friedrich Engels: Werke, Bd. 1. Berlin: Dietz, S. 378-391.

Meyer, J. W./Rowan, B. (1977): Institutionalized Organizations: Formal Structure as Myth and Ceremony. In: American Journal of Sociology 83 (2), S. 340-363. 
Morgan, G./Froud, J./Quack, S./Schneiberg, M. (2011): Capitalism in crisis: organizational perspectives. In: Organization 18 (2), S. 147-152.

Nace, T. (2005): Gangs of America. The rise of corporate power and the disabling of democracy. Berrett-Koehler, San Francisco.

Neuberger, O. (1997): Individualisierung und Organisierung: die wechselseitige Erzeugung von Individuum und Organisation durch Verfahren. In: Ortmann, G./Sydow, J./Türk, K. (Hrsg.): Theorien der Organisation. Die Rückkehr der Gesellschaft. Opladen: Westdeutscher Verlag, S. 487-522.

Ortmann, G. (1984): Der zwingende Blick. Personalinformationssysteme - Architektur der Disziplin. Frankfurt/Main, New York: Campus.

Ortmann, G. (2008): Organisation und Welterschließung. Dekonstruktionen, 2. Auflage. Wiesbaden: Springer VS.

Ortmann, G./Sydow, J. (2017): Dancing in chains: Creative practices in/of organizations. In: Organization Studies. Online First [DOI: 10.1177/0170840617717096].

Ortmann, G./Sydow, J./Windeler, A. (1997): Organisation als reflexive Strukturation. In: Günther O./Sydow, J./Türk, K. (Hrsg.): Theorien der Organisation. Die Rückkehr der Gesellschaft. Opladen: Westdeutscher Verlag, S. 315-354.

Parker, M. (1993). Life after Jean-Francois. In: Hassard, J./Parker, M. (Hrsg.), Postmodernism and organizations (S. 204-212). London: Sage.

Parker, M. (2002a): Against management. Organization in the age of managerialism. Cambridge: Polity Press.

Parker, M. (2002b): Utopia and the organizational imagination: eutopia. In: Parker, M. (Hrsg.), Utopia and organization. Oxford: Blackwell (Sociological review monographs), S. 217-224.

Parker, M./Cheney, G./Fournier, V./Land, Ch. (Hrsg.) (2014a): The Routledge companion to alternative organization. London: Routledge.

Parker, M./Cheney, G./Fournier, V./Land, Ch. (2014b): Introduction. In: Parker, M./Cheney, G./Fournier, V./Land, Ch. (Hrsg.), The Routledge companion to alternative organization. London: Routledge, S. 1-41.

Parker M, Cheney G, Fournier V, Land Ch (2014c): Horizons of Possibility: Challenge, CoOptation and Transformation. In: Parker M, Cheney G, Fournier V, Land C (Hrsg) The Routledge companion to alternative organization. Routledge, London, S 359-372.

Parker, M./Fournier, V./Reedy, P. (2007): The dictionary of alternatives. Utopianism and organization. London: Zed Books.

Raffnsøe, S./Gudmand-Høyer, M./Thaning, M. S. (2016): Foucault's dispositive: The perspicacity of dispositive analytics in organizational research. In: Organization 23 (2), S. 272-298.

Reedy, P./Learmonth, M. (2009): Other Possibilities? The Contribution to Management Education of Alternative Organizations. In: Management Learning 40 (3), S. 241-258.

Romero, J. M. (Hrsg.) (2014): Immanente Kritik heute. Grundlagen und Aktualität eines sozialphilosophischen Begriffs. Bielefeld: transcript. 
Rosa, H. (2009): Kritik der Zeitverhältnisse. Beschleunigung und Entfremdung als Schlüsselbegriffe der Sozialkritik. In: Jaeggi, R./Wesche, T. (Hrsg.): Was ist Kritik? Frankfurt/Main: Suhrkamp, S. 23-54.

Röttgers, K. (2004): Kritik. In: Brunner, O./Conze, W./Koselleck, R. (Hrsg.): Geschichtliche Grundbegriffe, Bd. 3. 8 Bände. Stuttgart: Klett-Cotta, S. 651-675.

Saar, M. (2007): Genealogie als Kritik. Geschichte und Theorie des Subjekts nach Nietzsche und Foucault. Frankfurt/Main, New York: Campus.

Saar, M. (2009): Genealogische Kritik. In: Jaeggi, R./Wesche, T. (Hrsg.): Was ist Kritik? Frankfurt/Main: Suhrkamp, S. 247-265.

Sarasin, P. (2006): Geschichtswissenschaft und Diskursanalyse. Frankfurt/Main: Suhrkamp.

Sloterdijk, P. (1983): Kritik der zynischen Vernunft. Frankfurt/Main: Suhrkamp.

Sontag, S. (2003): Das Leiden anderer betrachten. München, Wien: Hanser.

Starbuck, W. H. (1981): A trip to view the elephants and rattlesnakes in the garden of Aston. In: Van de Ven, A.H./Joyce, W.F. (Hrsg.): Perspectives on Organization Design and Behavior. New York: John Wiley \& Sons, S. 167-198.

Thompson, P./McHugh, D. (2009): Work organisations: a critical approach, 4. Auflage. Basingstoke: Palgrave Macmillan.

Türk, K. (1989): Neuere Entwicklungen in der Organisationsforschung. Ein Trend-Report. Stuttgart: Enke.

Türk, K. (1993): Politische Ökonomie der Organisation. In: Alfred Kieser (Hrsg.): Organisationstheorien, 1.Auflage. Stuttgart: Kohlhammer, S. 297-331.

Türk, K. (1995): „Die Organisation der Welt"“. Herrschaft durch Organisation in der modernen Gesellschaft. Opladen: Westdeutscher Verlag.

Türk, K. (1997): Organisation als Institution der kapitalistischen Gesellschaftsformation. In: Ortmann, G./Sydow, J./Türk, K. (Hrsg.): Theorien der Organisation. Die Rückkehr der Gesellschaft. Opladen: Westdeutscher Verlag, S. 125-176.

Vaughan, D. (1999): The dark side of organizations: Mistake, misconduct, and disaster. In: Annual Review of Sociology 25 (1), S. 271-305.

Walzer, M. (2000): Mut, Mitleid und ein gutes Auge. Tugenden der Sozialkritik und der Nutzen von Gesellschaftstheorie. In: Deutsche Zeitschrift für Philosophie 48 (5), S. 709-718.

Weiskopf, R. (2003): Management, Organisation, Poststrukturalismus. In: Weiskopf, R. (Hrsg.): Menschenregierungskünste. Anwendungen poststrukturalistischer Analyse auf Management und Organisation. Wiesbaden: Westdeutscher Verlag, S. 9-33.

Wesche, T. (2009): Reflexion, Therapie, Darstellung. Formen der Kritik. In: Jaeggi, R./Wesche, T. (Hrsg.): Was ist Kritik? Frankfurt/Main: Suhrkamp, S. 193-220.

Wolf, H. (1999): Arbeit und Autonomie. Ein Versuch über die Widersprüche und Metamorphosen kapitalistischer Produktion. Münster: Westfälisches Dampfboot.

Young, N. (1977): An infantile disorder? The crisis and decline of the New Left. Westview Press, Boulder, Colorado.

Zanoni, P./Contu, A./Healy, S./Mir, R. (2017): Post-capitalistic politics in the making: The imaginary and praxis of alternative economies. In: Organization 24 (5), S. 575-588.

Žižek, S. (1989): The sublime object of ideology. London: Verso. 
Žižek, S. (2009): Auf verlorenem Posten. Frankfurt/Main: Suhrkamp. 
Autorenangaben:

PD Dr. Ronald Hartz

Frühlingstrasse 12

01099 Dresden

E-Mail: ronald.hartz@wirtschaft.tu-chemnitz.de 